\title{
LAS PROHIBICIONES CONTRA LA MAFIA EN ITALIA
}

\section{PROHIBITIONS AGAINST THE MAFIA IN ITALY}

\author{
Vittoria Berlingò \\ Università degli Studi di Messina (Messina, Itália)
}

Recebimento: 5 dez. 2019

Aceitação: 4 mar. 2020

\begin{abstract}
Como citar este artigo / How to cite this article (informe a data atual de acesso / inform the current date of access):
BERLINGÒ, Vittoria. Las prohibiciones contra la mafia en Italia. Revista da Faculdade de Direito UFPR, Curitiba, PR, Brasil, v. 64, n. 3, p. 55-73, set./dez. 2019. ISSN 2236-7284. Disponível em: https://revistas.ufpr.br/direito/article/view/70558. Acesso $\quad$ em: $11 \quad$ mar. $2020 . \quad$ DOI:
\end{abstract} http://dx.doi.org/10.5380/rfdufpr.v64i3.70558.

\section{RESUMEN}

El documento se centra en los instrumentos contra las actividades de las organizaciones de la mafia, especialmente los "informazioni antimafia interdittive”, examinando las principales características de dichas medidas, los conflictos entre ellas y el principio de legalidad. Examinadas las principales disposiciones legislativas y decisiones judiciales sobre el tema, si pretende sistematizar todas las herramientas disponibles para el alcalde. El objetivo de este artículo es proponer un marco analítico que combine mejor las razones de las medidas de prevención y el principio de legalidad dentro de las políticas contra las actividades de las organizaciones de la mafia para evaluar la oportunidad de las experiencias con enfoque integrado, basándose tanto en la teoría como en la evidencia empírica ofrecida por los tribunales de decisiones. Secundariamente, describimos las manifestaciones del "efecto escalofriante" con respecto al "tipeo sombrío" de estas medidas, que se relacionan con el desarrollo social y económico en Italia. También mostramos cómo la intersección de las medidas de interdicción y reemplazo extraordinario en sus manifestaciones caracterizan los diferentes regímenes de integración. La propuesta de enfoques integrados se apoya en la doctrina contraria a la escoja de liderar una batalla contra la dinámica empresarial y económica patológica con actos de apuración, que tienen la mera función de interdicción al acceso al mercado. La nueva ley administrativa de “emergencias criminales" visa formas de intervención con el objetivo de traer el mercado a la legalidad para proteger la "posibilidad" de un ejercicio libre, verdadera y genuinamente de todas las iniciativas de la "economía legal”.

\section{PALABRAS CLAVE}

Fenómenos de infiltración mafiosa. Documentación antimafia. Ley de prevención. Tipificación "sombría”. Efecto de enfriamiento.

\section{ABSTRACT}

This document focuses on the instruments against the mafia organizational activities, especially the "informazioni antimafia interdittive", examining the characteristics of such measures, the conflicts within them, and with the legality principle. Once examined the main legal dispositions and judicial decisions about the subject, a systematization of the available for the mayor can be developed. The objective of this article is to propose an analytic framework able to combine the reasons behind preventive measures with the legality principle within the politics against the mafia organizational activities in order to evaluate the opportunity of such experiences with an integrated focus, based both 
in theory and empirical evidence offered by court decisions. Secondly, we describe the "chilling effect" derived from the "shady typing" of such measures, that are related with the social and economic development of Italy. We also demonstrate how the intersection of interdiction measures and extraordinary replacement in its manifestations characterize different integration regimes. The proposal of integrated focus is sustained by the doctrine opposed to the choice of leading a battle against a pathologically sick business and economic dynamic with polling acts, which possess the mere function of restricting access to market enterprises. The new administrative law of "criminal emergencies" intend to intervene with the intent to bring the market back to legality, protecting the "possibility" of a free - truly and genuinely - economical exercise of all the initiatives within a "legal economy”.

\section{KEYWORDS}

Mafia infiltration phenomena. Anti-mafia documentation. Prevention law. "Shady” legality. Chilling effect.

\section{INTRODUCCIÓN}

La cuestión de combatir el crimen organizado de tipo mafioso, quizás más que la corrupción, destaca una condición común en dos áreas del planeta, el sur de Europa y el sur de las Américas, que, sin embargo, requiere respuestas consistentes con el ecosistema legal de Cada una de estas áreas.

Fabrizio Fracchia (2018), en un editorial reciente de Federalismi, rastrea las prohibiciones italianas contra la mafia en una especie de medida, una expresión típica de la soberanía nacional y, por lo tanto, de una identidad cultural, en la que incluso el sistema legal brasileño solo puede inspirarse, sobre la base de un moción análoga a la reclamada en su momento por la estética carioca y descrita con la metáfora de la “deglutição”, mencionada en el Manifiesto de Antropófago por Oswald de Andrade (1890-1954) ${ }^{1}$.

Al continuar con el examen de la disciplina normativa de la documentación antimafia, trataré de resaltar los perfiles problemáticos, intentando aplicar, aunque con las distinciones apropiadas, a la vinculación del modelo antimafia italiano con el indicado por Nicola Gullo (2018), con efectividad expresión, como derecho administrativo de la 'emergencia penal'.

El documento se centra en los instrumentos contra las actividades de las organizaciones de la mafia, con especial atención a los llamados ‘informazioni antimafia interdittive’. El ensayo examina las principales características de dichas medidas y las razones de los conflictos entre ellas y el principio de legalidad. Después de examinar las principales disposiciones legislativas y decisiones judiciales sobre el tema, el artículo pretende sistematizar todas as herramientas disponibles para el alcalde, incluso medidas extraordinarias de reemplazo.

\footnotetext{
${ }^{1}$ Sobre el tema Losano (2015, p. 545 y siguientes).
} 
Más en detalle, el objetivo de este artículo es proponer un marco analítico que combine mejor las razones de las medidas de prevención y el principio de legalidad dentro de las políticas contra las actividades de las organizaciones de la mafia para evaluar mejor la oportunidad de las experiencias de un enfoque integrado.

Este marco está informado tanto por la teoría como por la evidencia empírica ofrecida por los tribunales de decisiones. Se basa en varias líneas de investigación y utiliza datos secundarios de varios estudios centrados en ejemplos de enfoques integrados en Italia.

Primero, se presenta una breve descripción general de las principales disposiciones legislativas para arrojar luz sobre la relación entre las mesureas de prevención, llamadas “informazioni antimafia interdittive”, y el principio de legalidad. En segundo lugar, describimos brevemente las manifestaciones del ‘efecto escalofriante' con respecto al 'tipeo sombrío' de estas mesureas, que se relacionan con el desarrollo social y económico en Italia. En tercer lugar, mostramos cómo la intersección de las dimensiones de las medidas de interdicción y reemplazo estraordinario en sus manifestaciones caracterizan la forma de los diferentes regímenes de integración.

En particular, la propuesta de enfoques integrados podría encontrar apoyo en la doctrina, particularmente de aquellos que formulan reservas sobre la escoja de liderar una batalla contra la dinámica empresarial y económica patológica o enferma, con simples actos de apuración, que tienen la mera función de interdicción al acceso al mercado. La nueva ley administrativa de 'emergencias criminales’ visa antes formas de intervención, mismo substitutivas, con el objetivo de traer el mercado de vuelta a la legalidad para proteger la 'posibilidad' de un ejercicio libre, verdadera y genuinamente de todas las iniciativas ligadas a la 'economía legal'.

\section{LA DOCUMENTACIÓN ANTIMAFIA (ART. 82 Y SIGUIENTES DEL DECRETO LEGISLATIVO N. 159 DE 2011)}

La representación del sistema de documentación antimafia, que se deriva de un primer examen del Libro II del decreto El código antimafia gira en torno a dos tipos de medidas: precisamente las comunicaciones antimafia y la información antimafia, como se menciona en el art. 84 de la d. LGS. n. 159 de $2011^{2}$.

Tanto el primero como el último se presentan como disposiciones constitutivas emitidas por los Prefectos (Órgano periférico del Ministerio del Interior que tiene funciones de representación

\footnotetext{
${ }^{2}$ Sobre los temas de compatibilidad constitucional establecidos por la legislación sobre documentación antimafia ver Scoca (2013).
} 
general del Gobierno del Estado en el territorio de la provincia o ciudad metropolitana) con respecto a la "confianza en la fiabilidad y la moralidad de un empresario”, que todas las administraciones públicas, organismos y empresas, sujetos a supervisión o control público, además de los concesionarios de obras públicas están obligados a solicitar antes de autorizar o aprobar al mismo contratista la estipulación de contratos y subcontratos relacionados con obras, servicios y suministros, o antes de emitir o permitir medidas con contenido extendido. Si este certificado plural es negativo, se requiere que las mismas administraciones adopten actos de decomiso, suspensión o prohibición con respecto a las actividades antes mencionadas.

Más específicamente, la legislación contra la mafia persigue el objetivo de la seguridad pública y la lucha contra el crimen organizado de tipo mafioso al referirse a dos perfiles de aplicación distintos:

- las relaciones de los particulares con las administraciones públicas (contratos públicos, concesiones y subsidios), para lo cual detecta el instrumento de información antimafia (mencionado en los artículos 90-95);

- las relaciones entre los propios particulares y, por lo tanto, cualquier actividad sujeta a autorización, concesión, autorización, registro en registros, o incluso a la notificación certificada de comienzo de actividad (cdscia) y a la provisión de consentimiento silencioso, para lo cual se detecta el instrumento de comunicación antimafia (artículos 87-89).

La colocación sistemática de la documentación contra la mafia en el Libro II del Código, subrayando la intención del legislador de considerar incluso la única documentación formalmente extraña contra la mafia en el aparato de medidas penales o parapinales y, por lo tanto, también desde el sistema de medidas de La prevención personal, regulada por separado por el Libro I del Código Anti-Mafia, ofrece más elementos útiles para especificar el alcance de la distinción mencionada anteriormente.

Las comunicaciones antimafia tienen un contenido restringido, ya que el supuesto de su emisión consiste en la certificación que a cargo de sujetos específicos, identificados por el art. 85 de la d. LGS. n. 159 de 2011, el Tribunal no ha dictado medidas definitivas de prevención personal. La comunicación antimafia fotografía así la cristalización de una situación de permeabilidad de la mafia contenida en una disposición jurisdiccional ahora definitiva.

La información contra la mafia, por otro lado, expresa un momento de marcada autonomía de evaluación por parte del prefecto, porque, al sopesar el riesgo de permeabilidad de la mafia de la empresa, la autoridad mencionada disfruta de amplios márgenes de discreción, como los hechos 
circunstanciales que detectan en orden para el ejercicio de esta función, pueden ignorar los resultados de investigaciones preliminares o procesos penales.

En este sentido, el legislador ha previsto una tipificación, por así decirlo, “sombría” de algunas situaciones sintomáticas de la infiltración de la mafia, incluida una serie de elementos heterogéneos.

Con respecto a esta tipificación, la jurisprudencia del Consejo de Estado italiano, con un fallo importante, el n. 1743 de 2016, trató de proporcionar indicaciones interpretativas claras y sin ambigüedades para permitir que las Prefecturas y los Tribunales Administrativos Regionales se orienten en este asunto. Por lo tanto, ha establecido que las situaciones sintomáticas tipificadas por el legislador, lejos de constituir un numerus clausus, asumen “diferentes formas y características según los tiempos, lugares y personas y escapan, debido a la insidiosa omnipresencia y mutabilidad, sobre todo en el plano social, del fenómeno de la mafia, a una clasificación precisa”.

\section{LA NATURALEZA DESPECTIVA DE LOS PODERES ADMINISTRATIVOS EN EL CONTEXTO DEL “DERECHO DE PREVENCIÓN" DE LOS FENÓMENOS DE INFILTRACIÓN MAFIOSA}

Según una primera lectura de las disposiciones ahora referidas a la lucha contra el crimen organizado, se plantea a sí misma como un “mero” asunto de orden público, al que las categorías legales y los recursos no muy diferentes a los que se encuentran en modelos de una tradición más que secular se les hace mirar de cerca. Podemos referirnos a la teoría antropológica subyacente a la ley para la represión del brigandage y los Camorrists en las áreas inaccesibles de 1863 y luego se reanudó en el t.u. en seguridad pública. Estas son medidas relacionadas con la presunta peligrosidad social del sujeto, basadas en el hecho objetivo de la comisión previa de un delito, y a menudo clasificables como un “caso de sospecha”, que de alguna manera corresponde al fenómeno de “sujeción penal” (sujeição criminal), identificado del sociólogo brasileño Michel Misse con respecto a las ‘figurinhas marcadas’ (como los favelados).

Al registrarse dentro de este marco teórico, la legislación contra la mafia parece haber tenido como objetivo fortalecer una “ley de prevención”, que evolucionó en un microsistema autónomo con respecto al penal, para enfrentar estados de excepción, con la configuración de poderes administrativos atípicos, extraordinario, ejercitable según criterios de emergencia, reclamable por el Estado debido a su competencia legislativa exclusiva en el asunto “orden público y seguridad” (art. 117, párrafo 2, letra h, Const.). 


\subsection{EXCEPCIONES CON RESPECTO AL PROCEDIMIENTO Y EL PROCESO}

Por lo tanto, la legislación administrativa antimafia invoca los requisitos de orden público para justificar el alcance despectivo de ciertas instituciones con respecto a las normas generales sobre el procedimiento administrativo, consagrado por l. n. 241 de 1990 y las leyes de reforma posteriores (considere, para todos y más recientemente, la Ley $\mathrm{N}^{\circ} 124$ de 2015), y la mitigación de las garantías procesales, dignas de protección solo si y en la medida en que sean compatibles con la protección de diferentes valores, de un rango superior, como los de orden público y de lucha contra el crimen organizado, que se destacan aquí.

También a nivel procesal, el alcance despectivo de los institutos es relevante tanto para la jurisdicción, atribuida al juez administrativo incluso cuando las disposiciones vinculadas emitidas por las administraciones ocurren en una fase avanzada de la relación contractual; ambos en términos de competencia, enraizados para las necesidades del proceso simultáneo, encabezado por el Tribunal Administrativo Regional, donde se encuentra la Prefectura que emitió la medida contra la mafia, también en el caso de que se cuestionen varios actos de revocación / retiro / decomiso administraciones individuales ubicadas en todo el territorio nacional; ambos, finalmente, en lo que se refiere al examen ritual y procesal, que es y sigue siendo el de la sentencia ordinaria, incluso cuando se apelan los actos de revocación y / o retirada, adoptados por las estaciones contratantes, sin que, por lo tanto, sea posible aplicar la disposición de los términos previstos el arte. 119, párrafo 2, c.p.a., para el rito de las licitaciones públicas.

\subsection{LAS REFLEXIONES DE LOS PERFILES DESPECTIVOS SOBRE EL TIPO DE UNIÓN EJERCIDA POR EL JUEZ ADMINISTRATIVO}

Con respecto a las repercusiones sobre el tipo de sindicato ejercido por el juez administrativo, debe tenerse en cuenta que, según la jurisprudencia administrativa, el sistema motivacional de información contra la mafia (ex se o en cuanto a la referencia a los actos de investigación) debe basarse en una representación general, atribuible a la autoridad prefectural, elementos de permeabilidad criminal que también pueden influir indirectamente en la actividad de la empresa.

Según el Consejo de Estado, es necesario evaluar el riesgo de contaminación de la mafia sobre la base del criterio consolidado de “más probable que no”, es decir, a la luz de una regla de juicio probabilístico, que bien puede complementarse con datos de experiencia común, que pueden deducirse de la observación de fenómenos sociales, como la mafia. Por esta razón, los elementos 
basados en la información contra la mafia también pueden no ser relevantes desde el punto de vista criminal o no ser objeto de procedimientos o juicios penales o, incluso a la inversa, pueden haber sido objeto de un juicio penal, con el resultado de la absolución o absolución.

Entre estos hechos la oración n. 1743 de 2016, mencionado anteriormente, procedió a ejemplificar una vasta serie de casos:

- la existencia de lazos emocionales que revelan una dirección “familiar” de la empresa, considerando también la estructura “familiar” o de clanes de las asociaciones de la mafia;

- los eventos corporativos anómalos, como valses en puestos sociales ocupados y cubiertos, en rotación, siempre por los mismos sujetos o por nominados;

- visitas repetidas a delincuentes que revelan una cercanía constante con las pandillas locales, relaciones de co-interés económico y asociaciones con delincuentes o empresas ya afectadas por medidas antimafia;

- el c.d. contigüidad adyacente y, obviamente, las condenas por el cc.dd. crímenes-espía.

En este sentido, es necesario tener en cuenta cómo, después de la oración n. 1743 de 2016, el porcentaje de cancelación de las medidas de prefectura es muy bajo y sustancialmente inferior al $10 \%$.

\section{LA APLICACIÓN DE INFORMACIÓN CONTRA LA MAFIA TAMBIÉN A ACTIVIDADES ECONÓMICAS ENTRE PARTICULARES}

Sin embargo, debe señalarse que la representación deducible de una primera lectura del Libro II del Código Anti-Mafia se vuelve más complicada si examinamos los cambios posteriores en el asunto.

Digamos de inmediato que los cambios regulatorios son la primera consecuencia de la superación de la dimensión dualista reconocida hasta ahora a la documentación antimafia, a favor de una categoría 'integral' de documentación antimafia, que, utilizando la expresión efectiva no por casualidad sugerida por los organizadores de la conferencia para el título de mi informe, también lo llamaré “descalificación antimafia”.

La referencia es al decreto correctivo n. 153 de 2014 que, para evitar el riesgo de infiltración de la mafia en la economía legal, independientemente de los contratos, concesiones o subsidios y, por lo tanto, de las relaciones de la empresa con la administración pública, también ha ampliado la efectividad interdictoria de la información antimafia a las autorizaciones y, por tanto, también a las 
relaciones entre particulares, superando emblemáticamente la rígida división tradicional de los efectos interdictorios, propia del binomio antimafia de comunicaciones / información ${ }^{3}$.

Esta es una eventualidad favorecida por el mismo decreto, que hizo operativa la base de datos nacional única de documentación administrativa (Artículo 98, párrafo 1, del Decreto Legislativo $\mathrm{N} \circ 159$ de 2011), permitiendo que la autoridad prefectural "tenga un conocimiento amplio y actualizado de la posición antimafia de una empresa” y de ser capaz de conocer otros elementos que lo lleven a investigaciones más serias sobre la posible permeabilidad de la mafia de la compañía y, por lo tanto, a la divulgación de información antimafia.

Así, se da un nuevo propósito a la disciplina en cuestión, la de la protección diferente y ahora autónoma de la economía legal. Un fallo reciente del Tribunal Constitucional de enero de 2018, al rechazar la cuestión de constitucionalidad planteada por el T.A.R. para Sicilia, sección separada de Catania, aclaró cómo “en el contexto de d. lg. n. 159 de 2011, y en base a la ley delegada n. 136 de 2010, nada que autorice [i] a pensar que el intento de infiltración de la mafia, establecido a través de la información interdictoria contra la mafia, no debe excluir también las actividades mencionadas en el art. 67, así como las relaciones contractuales con la administración pública, si el legislador lo ha establecido".

El pronunciamiento confirma lo que anteriormente fue apoyado por el Consejo de Estado, tanto en calidad de asesoría como judicial, a saber, que “el Estado no reconoce la dignidad y el estado de los operadores económicos, y ya no solo en las relaciones con la administración pública, a los sujetos condicionado, controlado, infiltrado y heterodireccionado por las asociaciones de la mafia”. Esta evaluación constituye un límite severo a la iniciativa económica privada, justificada, sin embargo, por la consideración de que el método de la mafia, por su propia razón de ser, constituye un “daño a la seguridad, la libertad y la dignidad humana” (párrafo segundo del artículo 41, Const.), ya en términos de relaciones entre individuos privados, en última instancia, realizando un límite al ejercicio de la misma propiedad privada.

La legislación antimafia más reciente, con la introducción del art. 89-bis de la d. LGS. n. 159 de 2011, por lo tanto, muestra que no quiere ignorar el hecho de que, entre la economía privada y la economía pública, existe, en el contexto actual de una economía globalizada, un tejido apretado para que ya no sea “pensable y posible contrarrestar la infiltración de ‘empresario' de la mafia y sus intereses en la [economía privada]”.

\footnotetext{
${ }^{3}$ Cons. St., Sección III, 8 de marzo de 2017, n. 1109, con una nota de Giustiniani y Fontana (2017, pp. 1414 y siguientes). 


\section{EFECTO DE DESCALIFICACIÓN}

Se deduce que la distinción por categorías (públicas / privadas) se retira, ya que ya no es importante enfatizar el campo de aplicación. La verificación del prefecto de la existencia de un “intento de infiltración de la mafia” se vuelve bastante decisiva, lo que, de hecho generalizado, es decisivo para la recurrencia del efecto de descalificación.

Antes de proceder a enmarcar el intento de infiltración de la mafia y a los perfiles problemáticos relacionados con él, se puede hacer referencia a la pronunciación adoptada, por la Asamblea Plenaria del Consejo de Estado italiano, el n. 3 de abril de 2018, sobre el alcance del efecto de descalificación, que acabamos de mencionar.

Las disposiciones del art. 94, según el cual las administraciones públicas "no pueden estipular, aprobar o autorizar los contratos o subcontratos, ni autorizar, liberar o, en ningún caso, permitir las concesiones y desembolsos”, lo especifica la asamblea administrativa suprema en el contexto de un caso relativo a sumas adeudado por la administración al empresario, a modo de compensación por el daño reconocido como juzgado formado después de la información. El asunto ofrece una oportunidad al Consejo de Estado para afirmar que la provisión de “descalificación antimafia” determina una forma de incapacidad ex lege, parcial y temporal, que impide que el sujeto, ya sea persona física o jurídica, tenga relaciones con el público Administración de conformidad con el art. 67 del Código Anti-Mafia.

\section{LA TIPIFICACIÓN ‘SOMBRÍA’ DEL 'INTENTO DE INFILTRACIÓN MAFIOSA’ Y LAS DESVIACIONES DEL C.D. EFECTO DE ENFRIAMIENTO}

Las descalificaciones contra la mafia, obtenidas ahora de las disposiciones combinadas de los artículos 89 bis y 91, injertados en una actividad de “contexto legal básico”, como la denominada. La 'economía legal', asumida, como se ha reiterado, con un activo legal que se debe preservar directa y autónomamente, resalta nuevos perfiles problemáticos que requieren que los intérpretes aborden en primer lugar un fenómeno muy relevante, el efecto o efecto escalofriante congelamiento, recientemente investigado por expertos en derecho penal (Manes, 2018, p. 2222).

La "tipicidad sombría”, que hemos visto, distingue el caso de “intento de infiltración de la mafia”, riesgos, para las derivas conectadas a una interpretación extensiva concreta y real y a una aplicación analógica de tendencia que se hace de esto, para comprimir excesivamente - o “congelar”, precisamente, los derechos subyacentes a la economía legal, como la libertad de iniciativa económica 
y el derecho a la propiedad, así como el derecho al trabajo (tanto del empresario como de los empleados).

Piense en esa especie de propiedad “transitiva” de la interdicción que se concreta en el c.d. "Efecto de contagio" que puede aplicarse tanto a la libertad de iniciativa económica como a la legislación laboral.

El primer evento ocurre cuando una empresa, con respecto a la cual no hay ningún elemento a considerar en riesgo, debido al hecho de comerciar con una empresa prohibida, sufre la interdicción, permaneciendo involucrado en el fenómeno de las descalificaciones en cascada: la empresa prohibido hace la segunda prohibición y así sucesivamente.

El segundo evento se puede inferir de dos eventos judiciales decididos, en diferentes momentos y con diferentes resultados, por dos tribunales de Calabria llamados a dictaminar sobre la legitimidad de algunos despidos.

El Tribunal de Locri, con un fallo de diciembre de 2009, estableció que el acto de retiro motivado por la simple preocupación de que la relación matrimonial con un presunto miembro de la mafia podría justificar la emisión de una disposición prefectoral que descalifique el negocio de la empresa. se remonta a una hipótesis de causa justa y ni siquiera a aquellas de razón subjetiva u objetiva justificada.

Por el contrario, el Tribunal de Crotone con una decisión hasta la fecha inédita a partir de noviembre de 2014, dictaminó en el sentido de la legitimidad de un despido ordenado por una empresa, con respecto a un empleado, justificó el despido después de la adopción por parte del autoridad prefectural de una lucha contra la mafia interdictoria promulgada debido a la "presencia masiva” entre los trabajadores de la compañía de sujetos vinculados a la cima del crimen organizado (Ranieri, 2015; Sciarrone, 2013; dalla Chiesa, 2012; Arlacchi, 2007).

\section{LA PROPORCIONALIDAD COMO CRITERIO-LÍMITE A LA EXTENSIÓN DEL TIPO LEGAL: CLAVES PARA DIFERENTES HIPÓTESIS RECONSTRUCTIVAS}

Los eventos mencionados anteriormente, como ejemplos de las consecuencias relacionadas con una extensión excesiva del tipo legal (y del efecto escalofriante) nos llevan a cuestionar la compatibilidad real de una disciplina antimafia, entendida como la máxima expresión de un 'derecho de prevención’, con respecto a las necesidades cambiadas, mientras tanto madurado.

Las respuestas de los intérpretes son de hecho diferentes. 
Por parte de quienes permanecen fieles a la idea de la suficiencia de un modelo antimafia conformado a poderes excepcionales y despectivos, la respuesta, que también se refleja en las direcciones del supremo juez administrativo siciliano ${ }^{4}$, se caracteriza por una crítica del componente discrecional reconocido en la evaluación del Prefecto, considerado como un mero expediente para justificar una revisión judicial débil por parte del juez administrativo, limitado al perfil de razonabilidad y congruencia. La propuesta es adherirse a la naturaleza auténtica de los actos de verificación propios de lo 'interdictorio', en cuyo contexto sería necesario dar la relevancia correcta a los hechos individuales como evidencia real, cada uno de los cuales, en busca de la verdad, no debería quedar abrumado por su evaluación general. Esto significa que deben determinarse hechos concretos, es decir, que alguien realmente intentó entrar en el gobierno corporativo, y no solo que ha tenido o ha tenido tratos con cualquiera de los gerentes de la compañía, y que también debe verificarse que el intento haya tenido éxito o tenga suficiente oportunidad de triunfar Estas consideraciones también se traducen en una graduación de los hechos circunstanciales, dado que, dado el diferente propósito de proteger la economía legal, se asigna mayor importancia a los contemplados por el cit. el arte. 91 - es decir, la trazabilidad de los flujos financieros, la política de contratación, la verificación de que los ejecutores materiales de las obras no son personas que no han sido indicadas - en lugar de aquellas limitadas solo a perfiles subjetivos, como la existencia de la relación pura y simple de cónyuges.

Según este enfoque interpretativo, la disposición interdictoria que se limita a afirmar que “uno o más familiares o amigos de la persona que solicita la certificación antimafia son 'mafia’ o ‘cercanos' a los sujetos de la mafia o vecinos ‘o' afiliado ‘a’ pandillas de la mafia ‘y / o' familias de la mafia”.

Esta es una parte importante de la jurisprudencia administrativa, adoptada más recientemente por Cons. St., Sección III, 27 de septiembre de 2018, n. 5547, que utilizaba la expresión de “familiarismo amoral” (aunque de origen sociológico), esencial para la correcta aplicación de la legislación sobre la prevención de la infiltración de la mafia, con la consecuencia de que, en caso de que la disposición prefectural interdictoria no respete Estos principios deben considerarse afectados negativamente.

Sin embargo, estos hallazgos no parecen ser incompatibles con los que conducen a una reconstrucción teórica diferente de la antimafia, como conocimiento especializado, dirigido no a la mera neutralización emergente de la capacidad de acción de la entidad mafiosa, sino también con el

${ }^{4}$ C.G.A. sentencias de 29 de julio de 2016, n. 247 y 3 de agosto de 2016, n. 257, con comentario Armao (2017), Mazzamuto (2013), Pergolizzi (2015), D’Angelo (2013) y Leotta (1997). 
propósito de reorganizar un déficit. Institución infiltrada. De acuerdo con estas pautas, los principios establecidos en el art. 97 de la Constitución, aplicada correctamente a los poderes administrativos antimafia, postulan una combinación virtuosa de interdicción y reestructuración, de fines represivos y conformativos o, de hecho, 'reeducativos' o 'reparativos', que pueden ser aquellos perseguidos por el Prefecto utilizando los poderes mencionados el arte. 32, párrafo 10, decreto legislativo n. 90 de 2012 sobre el tema de la lucha contra la corrupción, a saber: a) proporcionar, en casos de menor gravedad, el apoyo y la supervisión de la empresa (c.d. tutoría); b) ordenar la renovación, a través del reemplazo, de entidades corporativas y, en ausencia de adaptación dentro del plazo establecido, organizar la gestión extraordinaria y temporal de la empresa "limitada a la ejecución completa del contrato"; c) proporcionar directamente la gestión antes mencionada.

La confirmación de la validez de esta propuesta reconstructiva proviene del mismo decreto legislativo n. 153 de 2014, también intervino para coordinar el procedimiento de emisión de información contra la mafia con el de la aplicación de medidas administrativas, de gestión y de apoyo extraordinarias. En particular, en virtud del art. 94, párrafo 2-bis, decreto legislativo n. 159/2011, el Prefecto, habiendo adoptado el descalificador informe antimafia, verifica la existencia de los requisitos previos para la gestión del comisionado y, de ser así, informa al Presidente de la ANAC de inmediato. En última instancia, si durante la ejecución del contrato existe una información contradictoria contra la mafia, el Prefecto, después de haberla adoptado, debe verificar la existencia de las condiciones para la aplicación de las medidas extraordinarias de gestión temporal. Si, entonces, el Prefecto comunica a la estación contratante la inexistencia de las condiciones para la aplicación de tales medidas, corresponde a esta última decidir si rescindir el contrato o continuarlo de conformidad con el art. 94, párrafo 3, decreto legislativo n. 159/2011.

Las ideas reconstructivas importantes también pueden derivarse de la jurisprudencia administrativa.

De acuerdo con T.A.R. de Sicilia Catania Sección I, 20 de agosto de 2018, n. 1718, T.A.R. de Sicilia Palermo Sección I, 23 de julio de 2018, n. 1638 y, antes de eso, T.A.R. de Campania Nápoles, Sección I, n. 1933 de 26 de marzo de 2018, n. 1017 y n. 1019 de 2018,

\footnotetext{
la fórmula “elástica” adoptada por el legislador al regular la divulgación interdictoria contra la mafia sobre una base básica muestra la ponderación razonable entre el interés privado en el libre ejercicio de la actividad empresarial y el interés público en salvaguardar el sistema social económico de las contaminaciones de la mafia, donde la primera, como no está específicamente protegida por el CEDH o atribuible a la esfera de los derechos constitucionales inviolables, demuestra ser recesiva con respecto a la segunda, ya que está vinculada a las demandas preeminentes de defender el orden contra la acción antagónica del crimen organizado.
} 
Las resoluciones a las que se hace referencia, con las referencias contenidas en ellas, merecen especial atención porque, en una inspección más cercana, los jueces administrativos antes mencionados, al proceder con una interpretación 'adaptativa', es decir, conforme a la Constitución, de las disposiciones bajo examen, juzgan de acuerdo con el principio de La legalidad es un sistema de contraste con el crimen más articulado que el esencialmente dirigido a la mera neutralización de la capacidad de actuar de la institución infiltrada y a la consecuencia inefable de la ruptura de cualquier relación con la administración pública, ambos objetivos ya mejorados por el marco propuesto por el Consejo de Estado en su Asamblea Plenaria no. 3 de $2018^{5}$.

Los tribunales administrativos regionales se mueven, en primer lugar, de la recuperación del principio de la tipicidad del caso, señalando que la evaluación de la autoridad prefectural debe llevarse a cabo mediante la adquisición de elementos precisos de evidencia, con el corolario de que su adquisición debe llegar a resaltar un claro, completo y convincente del peligro de condicionamiento por el crimen organizado, para inducir, al mismo tiempo, al juez administrativo a un examen igualmente exhaustivo de estos elementos, individualmente y en su conexión íntima, incluso con un uso más valiente de la investigación preliminar ${ }^{6}$.

Esta es una directriz tomada y profundizada por la sentencia del 9 de julio de 2018, n. 385, del Consejo de Justicia Administrativa de Sicilia, según el cual es necessário

\footnotetext{
${ }^{5}$ Sobre esta última decisión, ver, para todos, Mazzamuto (2019).

${ }^{6}$ De acuerdo con T.A.R. Sección Reggio Calabria, 5 de febrero de 2018, n. 61, en materia de "negativa a registrar al solicitante en las listas de proveedores, prestadores de servicios y artistas intérpretes o ejecutantes no sujetos a intentos de infiltración de la mafia, según el art. 1, párrafo 52, de la Ley no. 190/2012, dado que el poder ejercido por la Autoridad de la Prefectura es una expresión de la lógica de anticipar el umbral de la defensa social, la medida de descalificación no debe necesariamente estar relacionada con las investigaciones en un caso penal definitivo sobre la existencia de la contigüidad de la empresa con organizaciones criminales, y, por lo tanto, del condicionamiento real de la actividad empresarial, pero puede estar respaldado por elementos sintomáticos y circunstanciales a partir de los cuales se evidencia el peligro de interferencia del crimen organizado en la actividad empresarial. No es suficiente demostrar la actualidad del intento de infiltrarse en la mafia, la mera relación con los sujetos que pertenecen al crimen organizado (ya que no es posible presumir automáticamente el condicionamiento de la empresa), pero la información contra la mafia debe indicar (además de relación familiar) también otros elementos a partir de los cuales es posible razonablemente y de acuerdo con una imagen circunstancial general deducir posibles vínculos entre los sujetos en cuya cuenta la autoridad de la prefectura ha identificado los prejuicios y la empresa ejercida por sus familiares. La información contra la mafia emitida por la Prefectura debe considerarse ilegítima si no aparece información de las Fuerzas Policiales para prefigurar algún tipo objetivo de riesgo de infiltración del crimen organizado en la empresa en cuestión, pero lo interdictorio está motivado solo por las relaciones de parentesco o amistad individual entre el empresario interesado y las personas sospechosas de vínculos o infiltraciones de la mafia sin verificación real de circunstancias concretas”. De nuevo T.A.R. Sección Reggio Calabria, 20 de agosto de 2018, n. 516: "La lucha contra la mafia interdictoria contra la sociedad cooperativa social, con el estatus de una escuela igual en el jardín de infantes, es ilegítima cuando la administración no ha producido actos o elementos concretos, más allá de las limitaciones parentales de los padres. Administrador único de la cooperativa, de la cual es posible, sobre la base del juicio de lo más probable que no lo haga, inferir la existencia de intentos de infiltración en la mafia o de lo que parece que la actividad comercial, que, debe recordarse, es una cooperativa sin fin con fines de lucro, puede, incluso indirectamente, facilitar actividades delictivas. El juicio de cesación de la peligrosidad social hecho por el Magistrado de Supervisión contra el sujeto afectado por la información antimafia es relevante y debe ser evaluado”.
} 
referirse siempre a una "conducta tipificada" o, como su equivalente, a uma "situación objetiva” (objetivamente perceptible) que la presupone (o que es un supuesto índice sintomático de la peligro de condicionamiento de la mafia), y agregó que el "método de investigación” que tiene como objetivo mejorar la llamada prevención "regla de lo más probable que no” puede acreditarse y usarse solo con la condición de que se combine con la “regla de máxima fiabilidad de la inducción” [...] cuanto más resumida sea la planta, menor será la fiabilidad de la juicio probabilístico [...] en ausencia de evidencias que acrediten la existencia de una conducta objetivamente perceptible que - en consideración de id quod plerumque accidit - revele al menos la intención de condicionar las decisiones estratégicas de otras personas, el juicio de probabilidad expira en un juicio de mera posibilidad ${ }^{7}$.

Las pronunciaciones mencionadas anteriormente, como se mencionó anteriormente, resaltan un punto de partida reconstructivo adicional en la parte en la que argumentan la interpretación orientada constitucionalmente basada en otras disposiciones del Código Anti-Mafia sobre los poderes de la prefectura, como la posibilidad de revisar una interdicción previa en un sentido liberador (Artículo 91, quinto párrafo) $^{8}$, y el de organizar la gestión extraordinaria de la empresa contratista para la continuación de la relación contractual (Artículo 32, párrafo 10, de la ley anticorrupción), aunque en la cara, debe aclararse de inmediato, la posibilidad reconocido a la misma administración otorgante o contratante, de conformidad con el art. 94 del Código Anti-Mafia, para continuar la relación contractual "en caso de que el trabajo se esté completando o, en el caso del suministro de bienes y servicios considerados esenciales para la búsqueda del interés público, si el sujeto que lo suministra no es puede ser reemplazado rápidamente”.

La impresión es que precisamente esta última referencia a medidas sustitutivas permite que la autonomía de un sistema para combatir la delincuencia organizada se base en bases más modernas y avanzadas que las del derecho punitivo y el sistema sancionador: las razones originales y exclusivas del orden El público podría ser reinterpretado y adaptado a las razones actuales del desarrollo socioeconómico ${ }^{9}$.

\footnotetext{
${ }^{7}$ La decisión, mencionada en el texto, está en línea con los Cons. Giust. AMM. Sicilia, 29 de julio de 2016, n. 247 y 3 de agosto de 2016, n. 257, con comentarios de Armao (2017). Más en general, ver Mazzamuto (2016).

${ }^{8}$ Sobre el alcance de la disposición, ver T.A.R. Sección Reggio Calabria, 25 de julio de 2018, n. 454, según el cual "La sumisión al juicio penal por el delito de perturbación de la subasta, si representa una indicación reveladora de la actualidad del riesgo de infiltración de la mafia en el momento de emitir el interdictorio, con la consiguiente legitimidad del mismo interdictorio en aplicación del principio tempus regit actum, necesariamente debe ser reexaminado contra la llegada de una sentencia absolutoria para confirmar la actualidad del peligro o si persiste la relevancia negativa de la conducta descrita en las disposiciones en cuestión, teniendo en cuenta el camino argumentativo específico que ha llevado al juez penal a una decisión favorable para el sospechoso. Debe declararse la ilegitimidad de un informe de descalificación contra la mafia que, si bien menciona la sentencia absolutoria, no explica su indiferencia o irrelevancia con respecto a la presunta prevalencia de los hechos surgidos durante el proceso penal e incorporados en el cuerpo de la misma información". Además, como lo señaló T.A.R. Sección Reggio Calabria, 31 de julio de 2018, n. 475, la "disposición con contenido" liberador "emitida por la Prefectura competente después de una revisión de la información contra la mafia y su posterior cancelación en defensa propia no conduce a la falta de interés de la empresa que interviene en la apelación, teniendo efecto ex nunc, con el consiguiente permanencia de los efectos tempore medios producidos por la medida interdictoria impugnada”.

${ }^{9}$ Sobre el tema, recientemente, Astone (2018).
} 
En consecuencia, se debe delinear un complejo articulado de competencias, que parece evocar la categoría del sistema seccional, como el hecho principal del control público de las empresas privadas, directamente atribuible al segundo párrafo del art. 41 de la Constitución ${ }^{10}$; dentro de dicho orden seccional, las competencias se distribuirían de acuerdo con la dinámica de la coordinación utilizada adecuadamente en los estudios más recientes para explicar los fenómenos de gestión de 'emergencias' ${ }^{11}$, sin excluir, sin embargo, la relevancia dogmática subyacente a la institución de la gestión obligatoria ${ }^{12}$, incluso si el ser considerado obsoleto hoy.

Y de hecho, según la Comisión Especial del Consejo de Estado de 18 de junio de 2018, en la Asamblea n. 1567, las medidas sustitutivas ordenadas por el Prefecto deben entenderse

como un instrumento contractual de autoprotección previsto directamente por la ley, relativo solo al contrato (y por lo tanto a la realización de la obra pública, a la prestación del servicio o a la ejecución del suministro) y no a la gobernanza firme. La justificación de la regla sería (es decir) permitir la finalización del programa contractual en interés exclusivo de la administración otorgante, a través de la gestión del contrato bajo el régimen de "legalidad controlada”.

El comisionado de la compañía adjudicataria del contrato, dirigido a la ejecución controlada del contrato, es el resultado de una opción expresa (y alternativa) referida a la determinación de la prefectura, justificada por la prevalencia motivada de razones (específicas y obligatorias) de "utilidad pública” (normativas y relacionadas de manera abstracta) a la perspectiva “continuidad de funciones y servicios que no pueden diferirse para la protección de los derechos fundamentales” o a la “salvaguarda de los niveles ocupacionales” o incluso a la "integridad de los presupuestos públicos”.

De esta manera, el impacto autoritario en la relación de negociación se mide no solo en el nivel (subjetivo) de la terminación de los órganos y gerentes administrativos ordinarios, sino también en el nivel (objetivo) del deber esculpido de “completar” los servicios divididos negociados.

Hay quienes han planteado algunas dudas: las prefecturas deben evaluar la oportunidad de adoptar medidas anticorrupción y no, en lugar de las administraciones que se delegan naturalmente, incluso la oportunidad de continuar la relación ${ }^{13}$.

Sin embargo, el Consejo de Estado no parece excluir que los supuestos de estas dos evaluaciones, aunque no coincidan completamente, pueden superponerse, ya que el interés de que se

\footnotetext{
${ }^{10}$ La teoría de los arreglos seccionales se debe a Giannini (2003), y, posteriormente, Giannini (1992), hoy en Scritti, Vol. IX, Milano 2006, 125. Sobre el debate en la doctrina con referencia a esta controvertida reconstrucción, ver Merusi (1964); Merusi (1966); Nigro (1968), como, finalmente, mencionado en Travi (2018).

${ }^{11}$ Finalmente ver Croce (2018). Finalmente, más generalmente, Giani y D’Orsogna (2018).

${ }^{12}$ La referencia es a Silvestri (1941 y 1969). Para una profundización de las implicaciones adicionales de la reconstrucción teórica, ahora referida, ver Tigano (1992).

13 Tan Mazzamuto (2018).
} 
ocupa la administración podría caer dentro de los intereses referidos al cuidado de la prefectura, en virtud de una coordinación se resolverá en el sentido de una prevalencia de la competencia de la prefectura como una proyección de la dimensión “técnica” del interés público para salvaguardar el sistema socioeconómico.

Lo anterior podría justificarse por el hecho de que la decisión de la administración de continuar la relación de conformidad con el art. 94 El Código Antimafia, a pesar de lo interdictorio, no impide que se produzca una medida anticorrupción prefectural, con la consecuente educación de la primera con respecto a la segunda, ya que se deriva de la oración normativa "incluso si las condiciones establecidas en el art. 94, párrafo 3, del Decreto Legislativo 6 de septiembre de 2011, n. 159” (art. 32, Decreto Legislativo n. 90/2014, cit.). Por lo tanto, parece igualmente lógico considerar que la decisión de la administración de retirarse de la relación, como resultado de la interdicción, no puede evitar, incluso en este caso, la adopción de medidas de prefectura inesperadas en la dirección opuesta, lo que implicará la ineficacia de la retirada y la continuación de la relación.

Como lo subrayan los mismos Contras. Sección Estatal III, con la sentencia de 24 de octubre de 2018, n. 6052, mientras que para la legitimidad de la revocación no hay necesidad de ninguna razón particular, por el contrario, es el uso de hipótesis excepcionales de conformidad con el art. 94 párrafo 3 del Decreto Legislativo no. 159 de 2011, que la aplicación de medidas extraordinarias de conformidad con el art. 32 D.L. n. 90 de 2014, siempre necesitan una indicación precisa de la naturaleza extraordinaria y concreta de las razones de interés público en su base.

En el orden seccional esbozado, ni siquiera parece que la función reparadora sea defectuosa, que también se menciona con respecto a las medidas antimafia en general, si, como se indica en la oración n. 6423 de T.A.R. Sección de Campania Nápoles I, de 2 de noviembre de 2018, admisión al control judicial, de conformidad con el art. 34 bis, Decreto Legislativo no. 159 de 2011, según lo introducido por el art. 11, párrafo 1, Ley 17 de octubre de 2017, n. 161, ordenado cuando los peligros de la infiltración implican "facilitar ocasionalmente la actividad de las personas contra quienes se ha propuesto o aplicado una medida contra la mafia”, se vuelve a conectar para preservar, en la medida de lo posible, las realidades productivas que, especialmente en las áreas donde existen los fenómenos de asociación criminal más llamativos, puede remediar la ausencia de oportunidades de empleo creíbles.

En la perspectiva de la reconstrucción así propuesta, tal como lo subraya el TAR al que se refirió por última vez, es importante tener en cuenta que el control judicial no se plantea como una superación o más bien una forma de atenuación de la sentencia formulada por la Prefectura con la 
información, sino más bien como una "incipiente" del procedimiento que condujo a la adopción de lo interdictorio, asumiéndolo ${ }^{14}$.

\section{BREVES CONCLUSIONES}

La propuesta de poner en el sistema todas las herramientas disponibles para el Prefecto, incluidas medidas extraordinarias, es valorada por aquellos que tienen reservas sobre la elección de liderar una batalla contra dinámicas empresariales y económicas patológicas o enfermas con simples actos de verificación que tienen la mera función de prohibición del acceso al mercado: una opción, de hecho, atribuible a un modelo de derecho administrativo del siglo XIX. La nueva ley administrativa, la recientemente identificada en la ley administrativa de 'emergencias criminales', no es un derecho que pueda basarse en actos puros de prohibición, prohibición y ejecución hipotecaria en el mercado, sino que debe apuntar a formas de intervención, incluso sustituir a devolviendo el mercado a la legalidad. La institución de la ley interdictoria no puede, por lo tanto, considerarse aisladamente y, de hecho, sería más congruente y productivo que la administración de la prefectura, encargada de gobernar el territorio, ejerza sus poderes de orden considerando todas las potencialidades intrínsecas del ejercicio de esta función.

Si los dos perfiles, respectivamente, de represión y reestructuración, se consideran unicum de la misma 'función', esta última estaría en conformidad con la Constitución no solo por el aspecto de la legalidad de la provisión del poder de interdicción, sino también en virtud de la proporcionalidad, razonabilidad, no contradicción de la provisión del momento conformativosustitutivo subsiguiente, que sigue a la interdicción y se destaca como complementaria a la misma.

Creo que la búsqueda de este resultado más integral es requerida por la circunstancia de proporcionar un modelo de legislación contra la mafia que merezca ser apreciado más allá de los límites de nuestro sistema legal nacional, un sello de efectividad productiva y justicia sustancial que lo haga aún más digno de atención para el su uso en el indispensable y cada vez más desafiante contraste con el crimen transnacional de la mafia.

\footnotetext{
${ }^{14}$ En este punto ver T.A.R. Sección Reggio Calabria, 30 de octubre de 2018, n. 643: “[...] el control judicial no constituye una superación de la interdicción antimafia, pero de cierta manera confirma su existencia, con la adopción de un régimen en el que la iniciativa empresarial se puede reanudar por razones de libertad de iniciativa y garantía de empleo, siempre naturalmente en un régimen restrictivo de sujeción a control extraordinario. Dada la naturaleza del control judicial y la expectativa que se deriva de él, la mera suspensión de los efectos de lo interdictorio (destinado, como tal, a operar para relaciones futuras y no también para el pasado), ni siquiera es posible reconocer tal medida efectividad retroactiva, de la cual se reduce el derrocamiento automático de los actos de medio tiempo adoptados por la administración”.
} 


\section{REFERENCIAS}

Arlacchi, P. (2007), La mafia imprenditrice: dalla Calabria al centro dell'inferno, Milano: il Saggiatore, 2007.

Armao, G. (2017), "Brevi considerazioni su informativa antimafia e rating di legalità ed aziendale nella prevenzione delle infiltrazioni criminali nei contratti pubblici”, Giustamm - Rivista di Diritto Administrativo, No. 3, pp. 3-5.

Astone, F. (2018), “Interdittive antimafia e 'commissariamento’ delle imprese (avuto riguardo al settore degli appalti pubblici)”, Giustamm - Rivista di Diritto Administrativo, No. 7.

Croce, F. (2018), “L'emergenza quale criterio di legittimazione soggettiva del potere”, in Scritti in memoria di Antonio Romano Tassone, Napoli: Editoriale Scientifica, pp. 867-893.

D’Angelo, G. (2013), “La documentazione antimafia nel D.Lgs. 6 settembre 2011, n. 159: profili critici”, Urbanistica e Appalti, No. 3, pp. 256-267.

dalla Chiesa, N. (2012), L'impresa mafiosa: tra capitalismo violento e controllo sociale, Milano: Cavallotti University Press.

Fracchia, F. (2018), “Sovranismi, globalizzazione e diritto amministrativo: sull’utilità di un approccio dialogante e a più dimensioni”, Federalimi.it, No. 17.

Giani, L. y D’Orsogna, M. y Police, A. (2018), Dal diritto dell'emergenza al diritto del rischio, Napoli.

Giannini, M. S. (1949), "Istituti di credito e servizi di interesse pubblico”, Moneta e credito, Vol. 2, No. 5.

Giannini, M. S. (1992), “Gli ordinamenti sezionali rivisitati”, Studi in onore di Vignocchi, Modena.

Giustiniani, M. y Fontana, P. (2017), "La tutela nei confronti delle infiltrazioni mafiose nelle attività economiche”, Giurisprudenza Italiana, pp. 1414-1418.

Gullo, N. (2018), Emergenza criminale e diritto amministrativo: L'amministrazione pubblica dei beni confiscati, Napoli: Editoriale Scientifica.

Leotta, E. (1997), “I poteri certificativi del Prefetto quali strumenti di contrasto alla criminalità organizzata: inquadramento sistematico ed aspetti problematici”, Giustizia amministrativa, No. 2.

Losano, M. G. (2015), “Sociologia giuridica e storica, storia del diritto” e, in Brasile, “Antropofagia giuridica”, en Materiali per una storia della cultura giuridica, pp. 545-574.

Manes, V. (2018), “Dalla 'fattispecie' al 'precedente': appunti di 'deontologia ermeneutica’”, Cassazione penale, Vol. 58, No. 6, pp. 2222-2249.

Mazzamuto, M. (2013), “Lo scettro alla prefettocrazia: l’indefinita pervasività del sottosistema antimafia delle grandi opere e il caso emblematico della 'filiera'”, Diritto Economia, pp. 619-635. 
Mazzamuto, M. (2016), "Profili di documentazione amministrativa antimafia”, Giustamm - Rivista di Diritto Administrativo, No. 3, pp. 1-50.

Mazzamuto, M. (2018), “La revisione delle interdittive prefettizie tra prevenzione antimafia e salvataggio delle imprese”, Giurisprudenza Italiana, Vol. 10, pp. 2222-2230.

Mazzamuto, M. (2019), "Pagamento di imprese colpite da interdittiva antimafia e obbligatorietà delle misure anticorruzione”, Giurisprudenza Italiana, No. 1, pp. 157-164.

Merusi, F. (1964), Le direttive governative nei confronti degli enti di gestione, Milano: Giuffrè.

Merusi, F. (1966), “Servizio pubblico”, Novissimo Digesto Italiano, Vol. 12, pp. 215-220.

Nigro, M. (1968), Profili pubblicistici del credito, Padova: Giuffré.

Pergolizzi, L. (2015), “L’informativa antimafia e i sistemi di monitoraggio sulle grandi opere: il caso ‘EXPO Milano 2015’”, Istituzioni del Federalismo, pp. 223-250.

Ranieri, M. (2015), “Contrasto alla criminalità organizzata e diritto del lavoro: indifferenze, interrelazioni e cortocircuiti”, Lavoro e diritto, No. 2, pp. 375-396.

Sciarrone, R. (2013). “Impresa mafiosa”, Dizionario enciclopedico di mafie e antimafia, p. 318.

Scoca, F. G. (2013), “Razionalità e costituzionalità della documentazione antimafia in materia di appalti pubblici”, Giustamm - Rivista di Diritto Administrativo, No. 6, pp. 13-14.

Silvestri, E. (1941), Le gestioni coattive, Milano: Giuffrè.

Silvestri, E. (1969), “Gestioni coattive”, Enciclopedia del Diritto, Vol. 18, pp. 681-696.

Tigano, A. (1992), "Premesse per uno studio su pluralismo amministrativo e legittimazione a resistere”, in Scritti per Enzo Silvestri Offerti Dagli Allievi, Milano: Giuffré, pp. 552-594.

Travi, A. (2018), “Il diritto amministrativo e l'ordinamento giuridico di Santi Romano”, Diritto Pubblico, Vol. 24, No. 3, pp. 905-920. 